\title{
Protective effect of valproic acid on cultured motor neurons under glutamate excitotoxic conditions. Ultrastructural study
}

\section{Ewa Nagańska, Ewa Matyja, Anna Taraszewska, Janina Rafałowska}

Department of Experimental and Clinical Neuropathology, M. Mossakowski Medical Research Centre, Polish Academy of Sciences, Warsaw, Poland

\begin{abstract}
Amyotrophic lateral sclerosis (ALS) is a fatal neurodegenerative disorder that involves the upper and lower motor neurons and leads to the patient's death within 5 years after diagnosis. Approximately 2 per 100,000 people worldwide are affected every year. The only FDA-approved drug available for medical treatment is riluzole. It slows the disease progression and improves limb function and muscle strength for 3-4 months. Thus, looking for new therapeutic agents is a pressing challenge.

Valproic acid (VPA) is a short-chain fatty acid, widely used for the treatment of seizures and bipolar mood disorder. The beneficial effect of VPA has been documented in different neurodegenerative experimental models, including amyotrophic lateral sclerosis (ALS). The real mechanisms underlying numerous beneficial effects of VPA are complex, but recently it has been postulated that the neuroprotective properties might be related to direct inhibition of histone deacetylase (HDAC).

The aim of this ultrastructural study was to evaluate the beneficial effect of VPA on the spinal cord motor neurons (MNS) in a glutamate (GLU)-induced excitotoxic ALS model in vitro. It had been previously documented that chronic GLU excitotoxicity resulted in various MN injuries, including necrotic, apoptotic and autophagic modes of cell death. The present results demonstrated the neuroprotective properties of VPA associated with inhibition of apoptotic and autophagic changes of spinal MNs in a model of neurodegeneration in vitro.
\end{abstract}

Key words: amyotrophic lateral sclerosis, valproic acid, neuroprotection, ultrastructural study.

\section{Introduction}

Amyotrophic lateral sclerosis (ALS), known as Charcot's or Lou Gehrig's disease, is a fatal neurodegenerative disease, one of the heterogeneous group referred to as motor neuron diseases (MNDs). It primarily affects upper and lower motoneurons (MNs) of the cerebral cortex, brainstem and spinal cord, but surrounding glial cells, muscle cells, interneurons, and Renshaw inhibitory neurons might also be involved [5]. The generalized weakness and progressive muscle atrophy usually lead to death by respiratory muscle failure within five years after the disease onset [42]. Most ALS cases occur sporadically, while about $5-10 \%$ of them are inherited and classified as familial form of the disease [1]. So far more than 16 causative 
genes responsible for ALS/MNDs have been identified. Among them, $10-20 \%$ are probably caused by mutations in the gene encoding $\mathrm{Cu} / \mathrm{Zn}$ superoxide dismutase-1 (SOD1), one of the main free-radical scavenging enzymes that protect cells against oxidative stress [2,37].

Until now, there is no effective therapeutic intervention for ALS, but different potential therapeutic targets are widely discussed [48]. It has been recently suggested that acetylation and/or deacetylation play an important role in the pathogenesis of different neurological diseases, including ALS [26]. Thus, histone deacetylase (HDAC) inhibitors have proved to be promising treatment in the broadly defined neurodegeneration.

Valproic acid (VPA; 2-n-propylpentanoic acid) is widely used as an anticonvulsant and mood stabilizing drug. Although different results suggest that this agent may exhibit a neuroprotective effect, the mechanism of such protection is not yet clear. VPA may exert its beneficial effect on neuronal survival and synaptic plasticity by acting directly on neurons and indirectly through glial cells [27]. Being a simple fatty acid, VPA is a substrate for the fatty acid $\beta$-oxidation (FAO) pathway, which takes place primarily in mitochondria. Recently, it has been also demonstrated that VPA effectively inhibits HDAC [15], making it valuable for investigations into the therapeutic role of chromatin remodeling in various pathological states of the central nervous system.
The purpose of this study was to determine whether valproate exhibits efficient neuroprotection against neuronal damage caused by glutamate toxicity in the organotypic culture of rat's lumbar spinal cord.

\section{Material and methods}

The study was performed on organotypic cultures of lumbar spinal cord obtained from 8-day-old rat pups. The cultures were kept at $36.6^{\circ} \mathrm{C}$ in a medium consisting of $25 \%$ inactivated fetal bovine serum and 75\% DMEM (Dulbecco Modified Eagle's Medium) supplemented with glucose to a final concentration of $600 \mathrm{mg} \%$ and with antibiotics. The medium was changed twice a week. On the $10-14^{\text {th }}$ day in vitro (DIV), the well-differentiated cultures were subjected to 1) glutamate (GLU) alone in a concentration of $100 \mu \mathrm{M}$; 2) $100 \mu \mathrm{M}$ GLU with $10 \mu \mathrm{M}$ VPA; 3) $10 \mu \mathrm{M}$ VPA alone. The control cultures were grown in standard conditions.

After 3, 6 and 11 days, the cultures were processed for electron microscopic study. They were rinsed in a cacodylate buffer ( $\mathrm{pH} 7.2$ ), fixed in a mixture containing $0.8 \%$ formaldehyde and $2.5 \%$ glutaraldehyde for 1 hour, postfixed in 1\% osmium tetroxide, dehydrated in alcohols in graded concentrations and embedded in Epon 812. Ultrathin sections were counterstained with uranyl acetate and lead citrate and examined in a JEOL 1200 EX electron microscope.

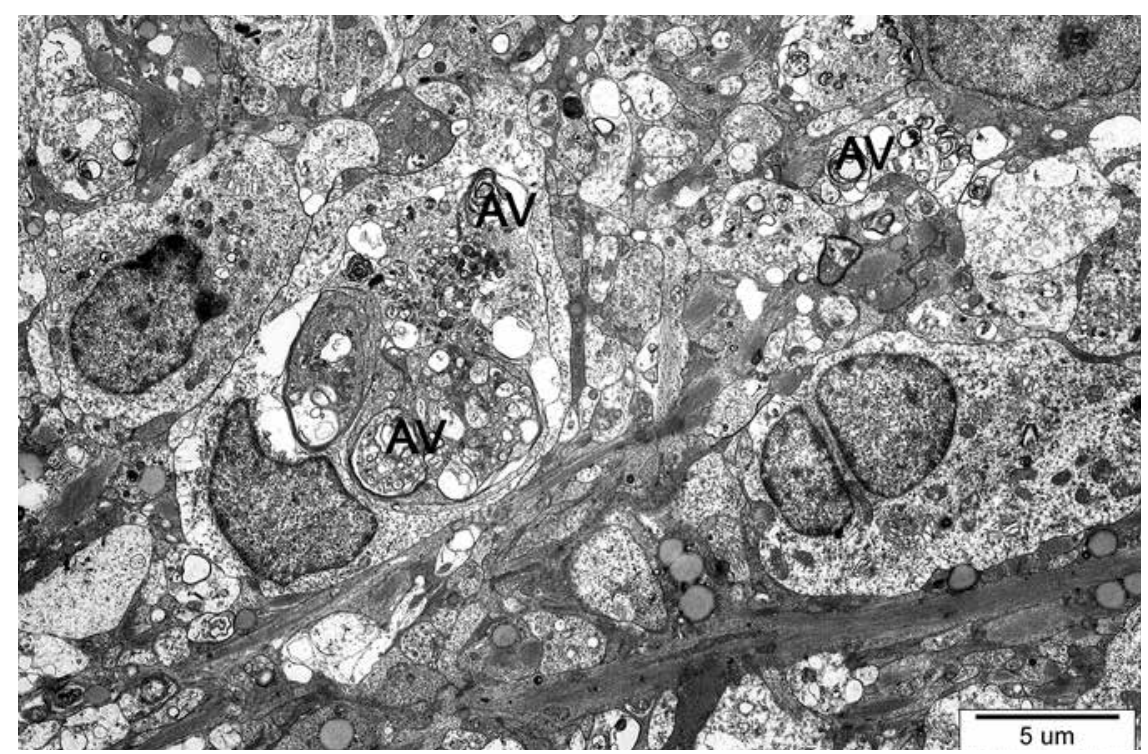

Fig. 1. Profiles of cellular processes and some neuronal cells with features of various degenerative changes, including numerous autophagic vacuoles (AV). Six days after $100 \mu \mathrm{M} \mathrm{GLU}$. 


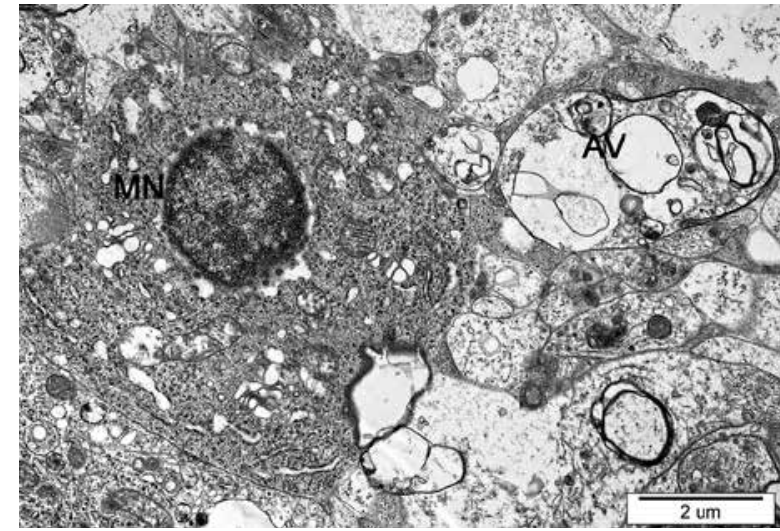

Fig. 2. Signs of apoptotic changes in motor neuron (MN), exhibiting condensed nuclear chromatin, increased electron density of cytoplasm and shrinkage of the whole cell body. Vesicular-autophagic changes (AV) seen in the vicinity of MN. Numerous damaged neuronal processes with signs of autophagy and destruction of organelles in surrounding neuropil. Eleven days after $100 \mu \mathrm{M}$ GLU.

\section{Results}

The spinal cord cultures exposed to GLU exhibited a range of advanced degenerative changes of MNs and destruction of neuronal processes in the surrounding neuropil. After 6 days of GLU exposure, the signs of various modes of cell death, including necrotic, apoptotic and autophagic changes, could be seen. Necrotic changes of MNs and their pro-

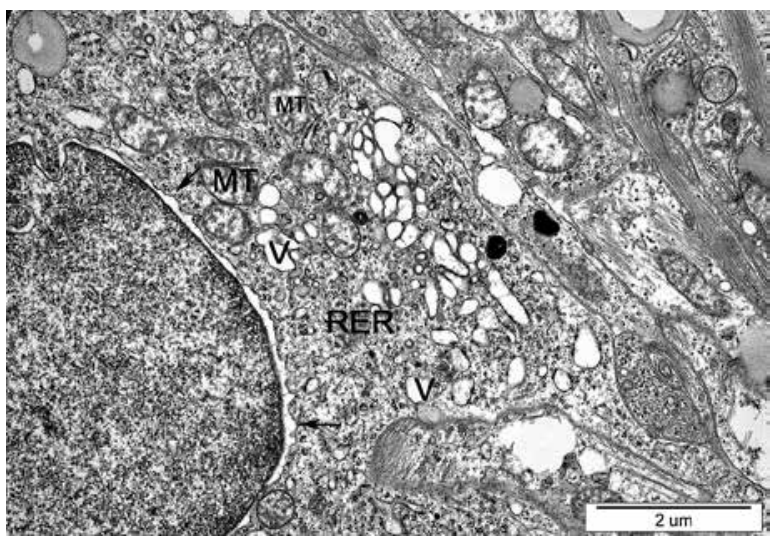

Fig. 4. Fragment of motor neuron exhibiting dispersed rough endoplasmic reticulum (RER), increased number of intracytoplasmic vesicles (V), distended perinuclear space (arrows) and slightly swollen mitochondria (MT). Eleven days after $100 \mu \mathrm{M} \mathrm{GLU}$.

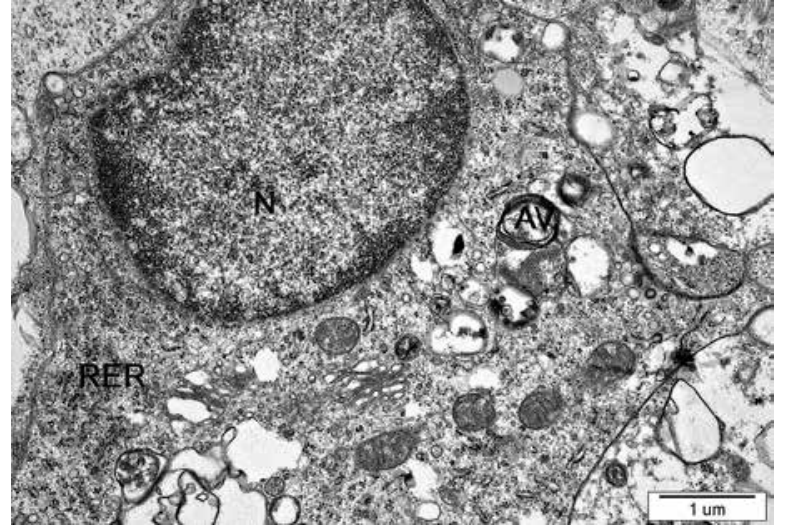

Fig. 3. Motor neuron with cytoplasm containing numerous autophagic vacuoles (AV) and dispersion of rough endoplasmic reticulum (RER). Nucleus ( $\mathrm{N}$ ) with condensed chromatin. Eleven days after $100 \mu \mathrm{M}$ GLU.

cesses were characterized by extensive swelling and total destruction of organelles. The apoptotic bodies within large autophagic vacuoles were seen (Fig. 1). After 11 days of exposure to GLU, many MNs displayed signs of apoptotic changes in the form of a shrinkage of the cell body and condensation of nuclear chromatin (Fig. 2). Moreover, numerous autophagic vacuoles in the cytoplasm of MNs and neuronal processes were observed (Figs. 2 and 3). Less severe damage of MNs includes disaggregation and loss of rough endoplasmic reticulum and the Golgi complex, increase of cytoplasmic microvesicles and swelling of mitochondria (Fig. 4).

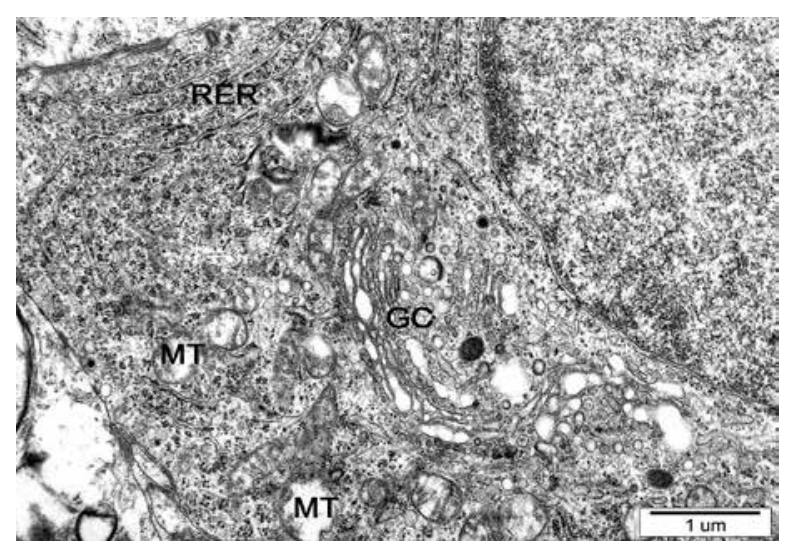

Fig. 5. Motor neuron with well-preserved nucleus and cytoplasm containing long, regularly arranged profiles of rough endoplasmic reticulum (RER), Golgi complex (GC), rich ribosomes and swollen mitochondria (MT). Three days after $100 \mu \mathrm{M} \mathrm{GLU}$ $+10 \mu \mathrm{M}$ VPA. 


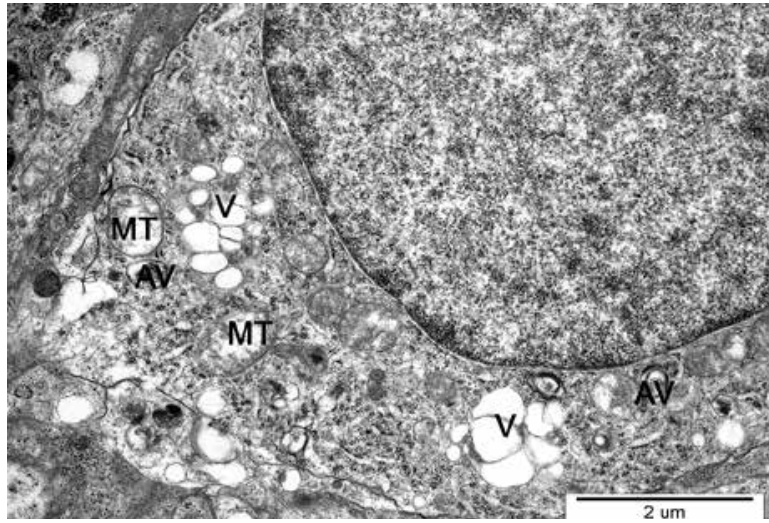

Fig. 6. Fragment of motor neuron with well-preserved nucleus and slight cytoplasmic abnormalities including presence of cytoplasmic vacuoles (V) with a few autophagic vacuoles (AV) and swollen mitochondria (MT). Three days after $100 \mu \mathrm{MGLU}$ $+10 \mu \mathrm{M}$ VPA.

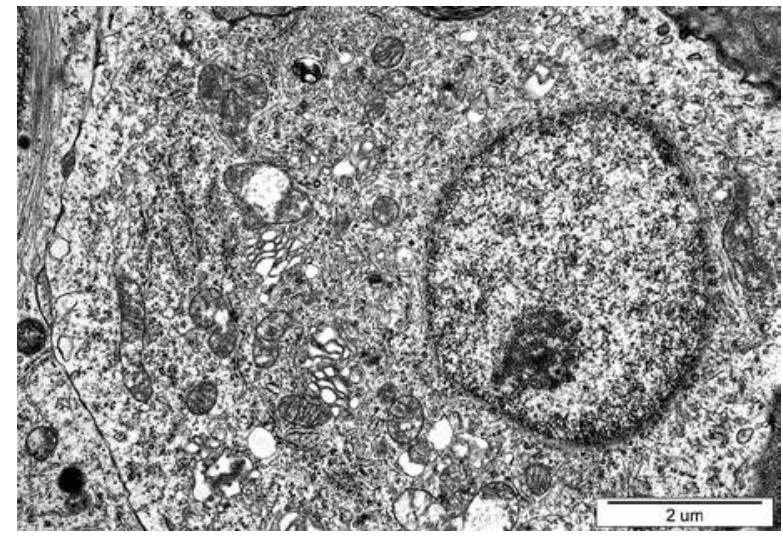

Fig. 8. Fragment of neuron, containing quite wellpreserved organelles and nucleus with distinct nucleolus and dispersed heterochromatin. Six days after $100 \mu \mathrm{M}$ GLU + $10 \mu \mathrm{M}$ VPA.

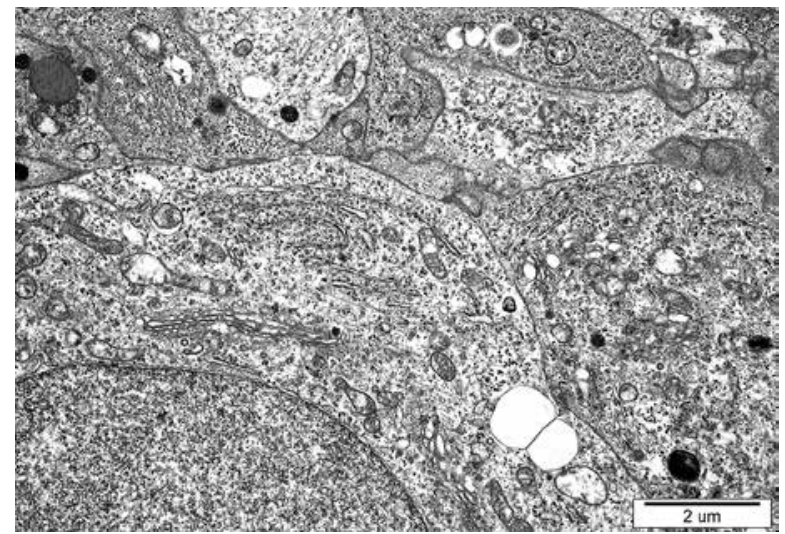

Fig. 10. Fragments of quite well-preserved neurons surrounded by neuropil with normal appearing neuronal and glial processes. Eleven days after $100 \mu \mathrm{M} \mathrm{GLU}+10 \mu \mathrm{M}$ VPA.

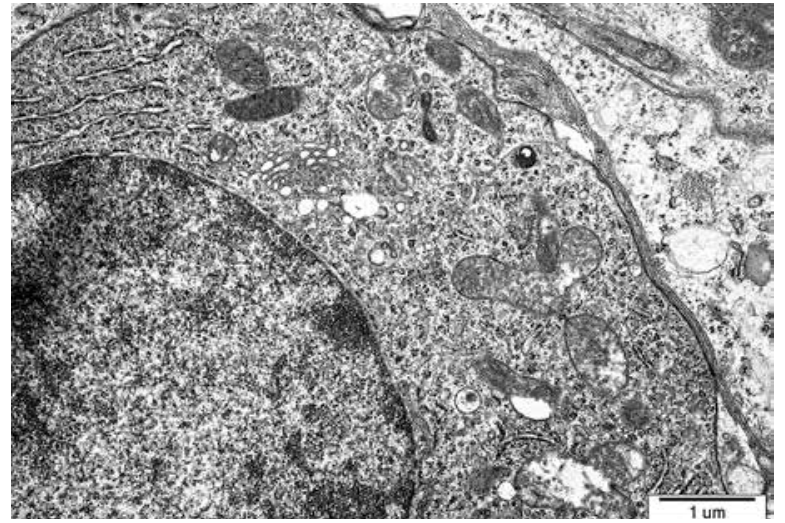

Fig. 7. Fragment of well-preserved motor neuron with normal organelles. Six days after 100 $\mu M G L U+10 \mu M$ VPA.

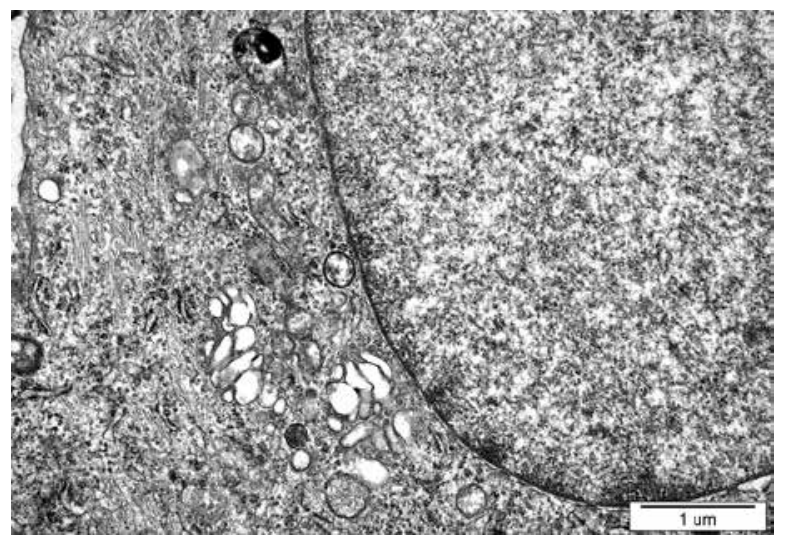

Fig. 9. Fragment of well-preserved motor neuron with large nucleus and cytoplasm containing normal organelles, microtubules and neurofilaments. Eleven days after $100 \mu \mathrm{M} \mathrm{GLU}+10 \mu \mathrm{M}$ VPA.

The treatment with VPA effectively prevents GLUinduced MN degenerative changes. Numerous wellpreserved large motor neurons could be identified after 3, 6 and 11 days of co-treatment of VPA and GLU. After 3 days of VPA + GLU exposure the neurons exhibited perikaryal cytoplasm containing long profiles of rough endoplasmic reticulum, a well-developed Golgi complex (Fig. 5), and sometimes slightly swollen mitochondria and small autophagic vacuoles (Fig. 6). Quite well-preserved MNs, exhibiting a large nucleus with dispersed chromatin, distinct nucleolus and cytoplasm, containing well-organized rough endoplasmic reticulum and Golgi complex, were still recognizable after 6 days (Figs. 7 and 8) and 11 days (Figs. 9 and 10) of VPA + Glu exposure. Numerous microtubules and 
neurofilaments in perikaryal cytoplasm were noticed (Fig. 9). The neuropil contained unchanged neuronal and glial processes (Fig. 10). Some large MNs exhibited only subtle ultrastructural abnormalities, such as slightly damaged mitochondria, focal cytoplasmic vacuolization and a few autophagic vacuoles. MNs with signs of apoptotic changes or autophagic degeneration could be seen only occasionally. Totally destroyed cells appeared sporadically.

\section{Discussion}

The mechanism underlying the pathogenesis of ALS remains unknown [28]. Genetic, molecular and environmental factors are believed to participate in the development of this progressive neurodegenerative process. Among various pathogenic factors, oxidative stress, mitochondrial dysfunction, apoptosis, glutamate excitotoxicity and proteasomal dysfunction are considered. However, the real cause of the disease, its natural history, classification, mechanism of progression and potential therapeutic targets are still under debate [13,29-31,35,36,41].

The cumulative evidence suggests that a mutation in Cu/Zn-superoxide dismutase (SOD1) protein contributes to the pathogenesis of familial ALS $[38,44]$. Dysfunction of neuronal mitochondria has been suggested to play an important role in MN degeneration [20]. The oxidative and endoplasmic reticulum (ER) stress $[16,17]$ and deregulation of the ER mitochondrial calcium cycle [22] are described as the most likely causes of motor neuronal death, but it is believed that a complex mechanism of multiple toxic pathways is implicated in the ALS onset and progression [32]. Glutamate-induced excitotoxicity is also considered as one of the possible pathophysiological factors of motor neuron death $[4,12]$.

Recently, a decreased histone acetylation level has been reported in the degenerating MNs in ALS experimental models [18]. Low histone deacetylase 6 (HDAC6) expression was reported at the onset and at the late stages of ALS in a mouse model [6]. Thus, it could be suggested that histone deacetylase inhibition may be a promising new therapeutic strategy for various neurodegenerative disorders, including motor neuron diseases (MNDs) $[8,10]$. As valproic acid (VPA), a short-chain fatty acid, effectively inhibits histone deacetylase [14,25] and delays apoptosis in degenerating neurons, it may be a good candi- date for studies on its therapeutic role in different neurodegenerative conditions $[3,19]$. Its therapeutic potential has been documented in various cellular and animal models of neurologic, neurodegenerative, and neuropsychiatric disorders [7]. Combined treatment with lithium and VPA delays onset of clinical symptoms, reduces neurological deficits and prolongs survival in the SOD1 mouse model of amyotrophic lateral sclerosis [11]. Co-treatment of cerebellar granular cell cultures with lithium and VPA induced synergistic neuroprotective effects against glutamate excitotoxicity in a time-dependent manner [24].

Histone proteins organize DNA into nucleosomes, which are regular repeating structures of chromatin. This organization is required for the efficient packaging of large amounts of eukaryotic genomic DNA [45]. Acetylation and deacetylation of histone proteins play an important role in various cellular events, including epigenetic regulation of transcription. Histone acetyltransferases (HATs) catalyze acetylation, whereas histone deacetylases (HDACS) enhance deacetylation. Therefore VPA, via HDAC inhibition, might potentiate gene expression and promote a more transcriptionally active chromatin conformation. Thus, VPA protects neurons from excitotoxicity through inhibition of HDAC activity and suppression of apoptotic neuronal death associated with nuclear accumulation of glyceraldehyde-3-phosphate dehydrogenase (GAPDH) [21]. It was also suggested that VPA exerts neuroprotective effects through changes in a variety of intracellular signaling pathways, including upregulation of $\mathrm{Bcl}-2$ protein with an antiapoptotic property [40]. Some results suggest that chronic treatment with valproate produce neuroprotection of cultured neuronal cells from damage caused by endoplasmic reticulum stress-mediated apoptosis [47] and oxidative stress [46]. It has been shown that combined treatment with histone deacetylase inhibitor and catalytic oxidant exerts additive neuroprotective effect in a transgenic mouse model of ALS [33]. Rouaux et al. [40] demonstrated that chronic VPA treatment in vivo prevented histone deacetylation in the spinal cord of symptomatic ALS mice. It delayed MN death and disease onset but did not significantly increase the mean lifespan of SOD1 (G86R) transgenic ALS mice and did not prevent distal pathology, i.e. neuromuscular denervation. However, despite the beneficial effect on MNs, VPA-treated animals died with 
the neuropathological features typical for ALS. These results indicate that beneficial effects of VPA might be related to other factors than only a strict neuroprotection. On the other hand, neuroprotection of lower MNs is not sufficient for clinical therapy of ALS, because it does not prevent neuromuscular denervation.

In the mouse ALS model, CREB-binding protein, a transcriptional coactivator with histone acetyltransferase activity, was specifically reduced in motor neurons of the lumbar spinal cord. Consistently, decreased histone acetylation levels were observed in degenerating motor neurons [39]. Numerous studies have reported the beneficial effects of HDAC inhibitors on different aspects of neurodegeneration. Although treatment with VPA was found to protect motor neurons against glutamate toxicity in an organotypic culture of spinal cord and the ALS mouse model [43], another study using the same G93A mouse model found that long-term dietary VPA administration protected motor neurons but did not significantly affect the animal lifespan [9]. Overall, these data indicate poor efficiency of HDAC in treatment for ALS therapy despite the neuroprotective efficiency of HDAC. The randomized sequential clinical trial evidenced that VPA, at one dosage corresponding to the therapeutic dose used in epilepsy, did not show a beneficial effect on survival and disease progression in patients with ALS [34]. It has implications for future trials in neurodegenerative diseases with various histone deacetylase inhibitors.

Our ultrastructural study documented the neuroprotective efficacy of VPA in an experimental model of neurodegeneration related to GLU excitotoxicity in vitro. GLU-induced excitotoxicity has been implicated in the pathophysiology of various neurodegenerative diseases, including ALS. It was shown that valproic acid protected cultured cerebellar granule cells against GLU-induced excitotoxicity with concomitant transcriptional activation and induction of $\alpha$-synuclein. This presynaptic protein was induced by valproic acid through histone deacetylase inhibition and participates in neuroprotection [23].

Our ultrastructural study evidenced that prevention of neuronal excitotoxic changes in vitro was related to inhibition of apoptosis and autophagy. The cultures subjected to GLU with VPA treatment exhibited well-retained parallel profiles of rough endoplasmic reticulum in the majority of motoneurons at 3 to 11 days after exposure. These results might sup- port previous data indicating that neuroprotective effects of VPA might act via suppressing ER stress-mediated apoptosis. It could suggest that this mechanism may cause the VPA-induced therapeutic effects in neurodegenerative processes. Additional studies are necessary to clarify this issue.

\section{Acknowledgments}

This study was supported by grant no. NN 401 014640 from the Ministry of Science and Higher Education of Poland and by statutory funds from the Mossakowski Research Institute, Polish Academy of Science.

\section{Disclosure}

Authors report no conflict of interest.

\section{References}

1. Ajroud-Driss S, Siddique T. Sporadic and hereditary amyotrophic lateral sclerosis (ALS). Biochim Biophys Acta 2015; 1852: 679-684.

2. Andersen PM, Sims KB, Xin WW, Kiely R, O’Neill G, Ravits J, Pioro E, Harati Y, Brower RD, Levine JS, Heinicke HU, Seltzer W, Boss M, Brown RH, Jr. Sixteen novel mutations in the Cu/Zn superoxide dismutase gene in amyotrophic lateral sclerosis: a decade of discoveries, defects and disputes. Amyotroph Lateral Scler Other Motor Neuron Disord 2003; 4: 62-73.

3. Biermann J, Grieshaber P, Goebel U, Martin G, Thanos S, Di Giovanni S, Lagreze WA. Valproic acid-mediated neuroprotection and regeneration in injured retinal ganglion cells. Invest Ophthalmol Vis Sci 2010; 51: 526-534.

4. Blasco H, Mavel S, Corcia P, Gordon PH. The glutamate hypothesis in ALS: pathophysiology and drug development. Curr Med Chem 2014; 21: 3551-3575.

5. Boillee S, Vande Velde C, Cleveland DW. ALS: a disease of motor neurons and their nonneuronal neighbors. Neuron 2006; 52: 39-59.

6. Chen S, Zhang XJ, Li LX, Wang Y, Zhong RJ, Le W. Histone deacetylase 6 delays motor neuron degeneration by ameliorating the autophagic flux defect in a transgenic mouse model of amyotrophic lateral sclerosis. Neurosci Bull 2015; 31: 459-468.

7. Chiu CT, Wang Z, Hunsberger JG, Chuang DM. Therapeutic potential of mood stabilizers lithium and valproic acid: beyond bipolar disorder. Pharmacol Rev 2013; 65: 105-142.

8. Chuang DM, Leng Y, Marinova Z, Kim HJ, Chiu CT. Multiple roles of HDAC inhibition in neurodegenerative conditions. Trends Neurosci 2009; 32: 591-601.

9. Crochemore C, Virgili M, Bonamassa B, Canistro D, PenaAltamira E, Paolini M, Contestabile A. Long-term dietary administration of valproic acid does not affect, while retinoic acid decreases, the lifespan of G93A mice, a model for amyotrophic lateral sclerosis. Muscle Nerve 2009; 39: 548-552. 
10. Echaniz-Laguna A, Bousiges O, Loeffler JP, Boutillier AL. Histone deacetylase inhibitors: therapeutic agents and research tools for deciphering motor neuron diseases. Curr Med Chem 2008; 15: 1263-1273.

11. Feng HL, Leng Y, Ma CH, Zhang J, Ren M, Chuang DM. Combined lithium and valproate treatment delays disease onset, reduces neurological deficits and prolongs survival in an amyotrophic lateral sclerosis mouse model. Neuroscience 2008; 155: 567-572.

12. Fulceri F, Ferrucci M, Lazzeri G, Paparelli S, Bartalucci A, Tamburini I, Paparelli A, Fornai F. Autophagy activation in glutamate-induced motor neuron loss. Arch Ital Biol 2011; 149: 101-111.

13. Gonzalez de Aguilar JL, Echaniz-Laguna A, Fergani A, Rene F, Meininger V, Loeffler JP, Dupuis L. Amyotrophic lateral sclerosis: all roads lead to Rome. J Neurochem 2007; 101: 1153-1160.

14. Gottlicher M. Valproic acid: an old drug newly discovered as inhibitor of histone deacetylases. Ann Hematol 2004; 83 Supp 1: S91-92.

15. Gottlicher M, Minucci S, Zhu P, Kramer OH, Schimpf A, Giavara S, Sleeman JP, Lo Coco F, Nervi C, Pelicci PG, Heinzel T. Valproic acid defines a novel class of HDAC inhibitors inducing differentiation of transformed cells. EMBO J 2001; 20: 6969-6978.

16. Ikawa M, Okazawa H, Tsujikawa T, Matsunaga A, Yamamura O, Mori T, Hamano T, Kiyono Y, Nakamoto Y, Yoneda M. Increased oxidative stress is related to disease severity in the ALS motor cortex: a PET study. Neurology 2015; 84: 2033-2039.

17. Ilieva EV, Ayala V, Jove M, Dalfo E, Cacabelos D, Povedano M, Bellmunt MJ, Ferrer I, Pamplona R, Portero-Otin M. Oxidative and endoplasmic reticulum stress interplay in sporadic amyotrophic lateral sclerosis. Brain 2007; 130: 3111-3123.

18. Janssen C, Schmalbach S, Boeselt S, Sarlette A, Dengler R, Petri S. Differential histone deacetylase mRNA expression patterns in amyotrophic lateral sclerosis. J Neuropathol Exp Neurol 2010; 69: 573-581.

19. Jeong MR, Hashimoto R, Senatorov VV, Fujimaki K, Ren M, Lee MS, Chuang DM. Valproic acid, a mood stabilizer and anticonvulsant, protects rat cerebral cortical neurons from spontaneous cell death: a role of histone deacetylase inhibition. FEBS Lett 2003; 542: 74-78.

20. Jiang Z, Wang W, Perry G, Zhu X, Wang X. Mitochondrial dynamic abnormalities in amyotrophic lateral sclerosis. Transl Neurodegener 2015; 4: 14.

21. Kanai H, Sawa A, Chen RW, Leeds P, Chuang DM. Valproic acid inhibits histone deacetylase activity and suppresses excitotoxicity-induced GAPDH nuclear accumulation and apoptotic death in neurons. Pharmacogenomics I 2004; 4: 336-344.

22. Lautenschlaeger J, Prell T, Grosskreutz J. Endoplasmic reticulum stress and the ER mitochondrial calcium cycle in amyotrophic lateral sclerosis. Amyotroph Lateral Scler 2012; 13: 166-177.

23. Leng Y, Chuang DM. Endogenous alpha-synuclein is induced by valproic acid through histone deacetylase inhibition and participates in neuroprotection against glutamate-induced excitotoxicity. J Neurosci 2006; 26: 7502-7512.

24. Leng Y, Liang MH, Ren M, Marinova Z, Leeds P, Chuang DM. Synergistic neuroprotective effects of lithium and valproic acid or other histone deacetylase inhibitors in neurons: roles of glycogen synthase kinase-3 inhibition. J Neurosci 2008; 28: 2576-2588.
25. Leng Y, Marinova Z, Reis-Fernandes MA, Nau H, Chuang DM. Potent neuroprotective effects of novel structural derivatives of valproic acid: potential roles of HDAC inhibition and HSP7O induction. Neurosci Lett 2010; 476: 127-132.

26. Liu D, Liu C, Li J, Azadzoi K, Yang Y, Fei Z, Dou K, Kowall NW, Choi HP, Vieira F, Yang JH. Proteomic analysis reveals differentially regulated protein acetylation in human amyotrophic lateral sclerosis spinal cord. PLoS One 2013; 8: e80779.

27. Monti B, Polazzi E, Contestabile A. Biochemical, molecular and epigenetic mechanisms of valproic acid neuroprotection. Curr Mol Pharmacol 2009; 2: 95-109.

28. Musaro A. Understanding ALS: new therapeutic approaches. FEBS I 2013; 280: 4315-4322.

29. Orsini M, Oliveira AB, Nascimento OJ, Reis CH, Leite MA, de Souza JA, Pupe C, de Souza OG, Bastos VH, de Freitas MR, Teixeira S, Bruno C, Davidovich E, Smidt B. Amyotrophic Lateral Sclerosis: New Perpectives and Update. Neurol Int 2015; 7: 5885.

30. Paez-Colasante X, Figueroa-Romero C, Sakowski SA, Goutman SA, Feldman EL. Amyotrophic lateral sclerosis: mechanisms and therapeutics in the epigenomic era. Nat Rev Neurol 2015; 11: 266-279.

31. Palomo GM, Manfredi G. Exploring new pathways of neurodegeneration in ALS: the role of mitochondria quality control. Brain Res 2015; 1607: 36-46.

32. Pasinelli P, Brown RH. Molecular biology of amyotrophic lateral sclerosis: insights from genetics. Nat Rev Neurosci 2006; 7 : 710-723.

33. Petri S, Kiaei M, Kipiani K, Chen J, Calingasan NY, Crow JP, Beal MF. Additive neuroprotective effects of a histone deacetylase inhibitor and a catalytic antioxidant in a transgenic mouse model of amyotrophic lateral sclerosis. Neurobiol Dis 2006; 22: 40-49.

34. Piepers S, Veldink JH, de Jong SW, van der Tweel I, van der Pol WL, Uijtendaal EV, Schelhaas HJ, Scheffer H, de Visser M, de Jong JM, Wokke JH, Groeneveld GJ, van den Berg LH. Randomized sequential trial of valproic acid in amyotrophic lateral sclerosis. Ann Neurol 2009; 66: 227-234.

35. Przedborski S, Mitsumoto H, Rowland LP. Recent advances in amyotrophic lateral sclerosis research. Curr Neurol Neurosci Rep 2003; 3: 70-77.

36. Ravits J. Focality, stochasticity and neuroanatomic propagation in ALS pathogenesis. Exp Neurol 2014; 262 Pt B: 121-126.

37. Rosen DR, Sapp P, O'Regan J, McKenna-Yasek D, Schlumpf KS, Haines JL, Gusella JF, Horvitz HR, Brown RH, Jr. Genetic linkage analysis of familial amyotrophic lateral sclerosis using human chromosome 21 microsatellite DNA markers. Am J Med Genet 1994; 51: 61-69.

38. Rotunno MS, Bosco DA. An emerging role for misfolded wildtype SOD1 in sporadic ALS pathogenesis. Front Cell Neurosci 2013; 7: 253.

39. Rouaux C, Jokic N, Mbebi C, Boutillier S, Loeffler JP, Boutillier AL. Critical loss of CBP/p300 histone acetylase activity by caspase-6 during neurodegeneration. EMBO I 2003; 22: 6537 6549.

40. Rouaux C, Panteleeva I, Rene F, Gonzalez de Aguilar JL, Echaniz-Laguna A, Dupuis L, Menger Y, Boutillier AL, Loeffler JP. Sodium valproate exerts neuroprotective effects in vivo through 
CREB-binding protein-dependent mechanisms but does not improve survival in an amyotrophic lateral sclerosis mouse model. J Neurosci 2007; 27: 5535-5545.

41. Rowland LP. Controversies about amyotrophic lateral sclerosis. Neurologia 1996; 11 Suppl 5: 72-74.

42. Rowland LP. Diagnosis of amyotrophic lateral sclerosis. J Neuro Sci 1998; 160 Suppl 1: S6-24.

43. Sugai F, Yamamoto Y, Miyaguchi K, Zhou Z, Sumi H, Hamasaki T, Goto M, Sakoda S. Benefit of valproic acid in suppressing disease progression of ALS model mice. Eur J Neurosci 2004; 20: 3179-3183.

44. Tafuri F, Ronchi D, Magri F, Comi GP, Corti S. SOD1 misplacing and mitochondrial dysfunction in amyotrophic lateral sclerosis pathogenesis. Front Cell Neurosci 2015; 9: 336.

45. Verdone L, Caserta M, Di Mauro E. Role of histone acetylation in the control of gene expression. Biochem Cell Biol 2005; 83: 344-353.

46. Wang JF, Azzam JE, Young LT. Valproate inhibits oxidative damage to lipid and protein in primary cultured rat cerebrocortical cells. Neuroscience 2003; 116: 485-489.

47. Wang X, Ma M, Teng J, Che X, Zhang W, Feng S, Zhou S, Zhang Y, Wu E, Ding X. Valproate Attenuates 25-kDa C-Terminal Fragment of TDP-43-Induced Neuronal Toxicity via Suppressing Endoplasmic Reticulum Stress and Activating Autophagy. Int J Biol Sci 2015; 11: 752-761.

48. Zhu Y, Fotinos A, Mao LL, Atassi N, Zhou EW, Ahmad S, Guan Y, Berry JD, Cudkowicz ME, Wang X. Neuroprotective agents target molecular mechanisms of disease in ALS. Drug Discov Today 2015; 20: 65-75. 